\title{
Influência de Leguminosas no Controle de Fitonematóides no Cultivo Orgânico de Alface Americana e de Repolho
}

\author{
Sylvia R. G. Moraes ${ }^{1}$, Vicente P. Campos ${ }^{1}$, Edson A. Pozza ${ }^{1}$, Anastácia Fontanetti ${ }^{2}$, Gabriel J. Carvalho ${ }^{2}$ \\ \& Cleber Maximiniano ${ }^{1}$
}

${ }^{1}$ Departamento de Fitopatologia; ${ }^{2}$ Departamento de Agricultura, Universidade Federal de Lavras, Cx. Postal 3037, CEP 37200-000, Lavras, MG, e-mail: sylmoraes@yahoo.com.br

(Aceito para publicação em 11/03/2006)

Autor para correspondência: Sylvia Raquel Gomes Moraes

\begin{abstract}
MORAES, S.R.G., CAMPOS, V.P., POZZA, E.A., FONTANETTI, A., CARVALHO, G.J. \& MAXIMINIANO, C. Influência de leguminosas no controle de fitonematóides em cultivo orgânico de alface americana e repolho. Fitopatologia Brasileira 31:188-191. 2006.
\end{abstract}

\section{RESUMO}

A demanda por produtos orgânicos é crescente devido às restrições ao uso dos agroquímicos sintéticos. Para verificar o efeito das plantas antagonistas no controle de fitonematóides na cultura da alface americana (Lactuca sativa) e do repolho (Brassica oleracea var. capitata), em cultivo orgânico, instalou-se um experimento na Universidade Federal de Lavras, no período de dezembro de 2001 a agosto de 2002. O delineamento experimental utilizado foi o de blocos casualizados em esquema fatorial 4 x 2, com três repetições. O primeiro fator foi constituído pelas leguminosas mucuna-preta (Stizolobium aterrimum), feijão-de-porco (Canavalia ensiformis), crotalária (Crotalaria juncea) em cultivo orgânico e por uma testemunha (vegetação espontânea) em cultivo convencional. O segundo, pelas culturas de alface americana cultivar Raider e repolho, cultivar Kenzan. Os tratamentos foram constituídos por todos em cultivo orgânico e testemunha (vegetação espontânea) em cultivo convencional. As populações de Meloidogyne spp. e de Helicothylenchus dihystera foram avaliadas empregando-se a técnica de Jenkins. As amostras de solo utilizadas para quantificar os nematóides foram retiradas aos 45 dias após o plantio das leguminosas; aos 30 e 60 dias após o plantio da alface americana e aos 30, 60 e 90 dias após o plantio do repolho. A incorporação das leguminosas mucuna-preta e crotalária, em cultivo orgânico, reduziu a população de Meloidogyne spp. em 42 e 51\%, respectivamente, nessas hortaliças. A leguminosa feijão-de-porco causou redução da população de nematóide apenas nas parcelas com repolho apos 90 dias.

Palavras-chave adicionais: planta antagonista, leguminosas, manejo de fitodoenças.

\begin{abstract}
Influence of legumes in the control of nematodes in organic crisphead lettuce and cabbage crops

The demand for organic products is increasing because of restrictions on the use of synthetic agrochemicals. In order to verify the effect of antagonistic plants on the control of phytonematodes in the culture of crisphead lettuce (Lactuca sativa) and cabbage (Brassica oleracea var. capitata), an experiment was installed at the Federal University of Lavras, between December 2001 and August 2002. The experimental design was of randomized blocks in a factorial scheme with three replications and four treatments. The treatments consisted of black velvet bean (Stizolobium aterrimum), jack bean (Canavalia ensiformis), sunn hemp (Crotalaria juncea) and a control plot with spontaneous vegetation. The populations of Meloidogyne spp. and Helicothylenchus dihystera were evaluated using the Jenkins technique. The soil samples used to quantify the nematodes were taken 45 days after seeding the leguminous plants, 30 and 60 days after seeding the crisphead lettuce and 30, 60 and 90 days after planting the cabbage. The incorporation of the black velvet bean and sunn hemp legumes reduced the population of Meloidogyne spp. by 42 and 51\%, respectively; in the area previously planted with jack bean, the nematode populations on cabbage were reduced at 90 days.
\end{abstract}

Additional keywords: antagonistic plants, leguminosae, plant disease management.

A agricultura orgânica fundamenta-se na melhoria da fertilidade do solo, tendo como princípio básico a aplicação de matéria orgânica, por meio de resíduos orgânicos vegetais ou animais, objetivando o equilíbrio biológico e a reciclagem de nutrientes (Darolt, 2002). A maior exigência dos consumidores por produtos isentos de agrotóxicos e que não foram geneticamente modificados e, portanto proporcionam menor impacto ambiental comparado ao sistema convencional são as principais razões para a expansão desse sistema de produção. No mundo, existem cerca de 23 milhões de hectares manejados com agricultura orgânica, desses aproximadamente $6 \%$ localizam-se no Brasil (Yussefi \& Willer, 2003).

Dentre as práticas utilizadas na agricultura orgânica, a adubação verde está entre as mais difundidas por promover melhorias nas características físicas, químicas e biológicas do solo, além de exercer importante efeito no manejo de doenças e de plantas invasoras. As leguminosas 
são geralmente as plantas mais utilizadas na adubação verde devido ao aumento do teor de nitrogênio no solo por fixação biológica e ainda por contribuir para reciclar nutrientes (Alcântara et al., 2000). Entre as leguminosas promissoras para a prática da adubação verde, no cultivo orgânico, destacam-se: a mucuna-preta (Stilozobium aterrimum Piper e Tracy), a crotalária (Crotalaria juncea L.) e o feijão-de-porco (Canavalia ensiformis D.C.), por serem plantas rústicas e de eficiente desenvolvimento vegetativo, adaptadas às condições de baixa fertilidade e de elevadas temperaturas (Pereira $e t$ al., 1992). Essas espécies também possuem a capacidade de reduzir a densidade populacional de nematóides parasitas de plantas (Bringel \& Silva, 2000). Andrade \& Ponte (1999) e Ribas et al. (2002), avaliando a utilização de $C$. juncea e $C$. spectabilis Roth. consorciadas com quiabeiro (Abelmoschus esculentus Moench.), observaram redução no número de galhas, formadas por fitonematóides, nas raízes do quiabeiro e conseqüente aumento na produtividade, porém em cultivo convencional.

Os nematóides causam sérios problemas na produtividade de hortaliças em diversas regiões do mundo. No Brasil, as perdas anuais variam de acordo com o manejo adotado pelo produtor (Zambolim et al., 1972). No entanto, a situação é mais crítica em sistema orgânico de produção, devido ao controle químico não ser permitido pelas normas de certificação, portanto, torna-se de extrema importância estudar técnicas alternativas para controlar fitonematóides, entre elas o emprego da adubação verde.

O presente trabalho teve como objetivo avaliar, no campo, a utilização de mucuna-preta, crotalária e feijão-deporco como tática no manejo de fitonematóides no cultivo orgânico da alface americana (Lactuca sativa L.) e do repolho (Brassica oleracea var. capitata L.).

O experimento foi conduzido no período de dezembro de 2001 a agosto de 2002 no Setor de Olericultura, do Departamento de Agricultura da Universidade Federal de Lavras, em Lavras, MG.

O delineamento experimental utilizado foi o de blocos casualizados em esquema fatorial 4 x 2 , com três repetições. $\mathrm{O}$ primeiro fator foi constituído pelas leguminosas mucunapreta, feijão-de-porco e crotalária e por uma testemunha (vegetação espontânea) e o segundo, pelas culturas de alface americana (cv. Raider) e repolho (cv. Kenzan). A parcela experimental teve a dimensão de $4 \times 3 \mathrm{~m}$.

O preparo do solo foi feito no sistema convencional, com aração e gradagem, no início do mês de dezembro. Em seguida, semearam-se a mucuna-preta e o feijão-deporco com espaçamento de $0,50 \mathrm{~m}$ entre linhas e $0,20 \mathrm{~m}$ entre plantas e a crotalária com $0,50 \mathrm{~m}$ entre linhas e $0,05 \mathrm{~m}$ entre plantas. Na testemunha as plantas daninhas não foram controladas, até o plantio das hortaliças.

As mudas das hortaliças foram produzidas em casade-vegetação coberta com plástico e as laterais fechadas com tela clarite. A semeadura foi feita em bandejas de isopor de 128 células contendo somente o substrato comercial Plantmax ${ }^{\circledR}$.
Noventa dias após a semeadura, as leguminosas foram roçadas e incorporadas ao solo. Um mês após a incorporação, o solo foi gradeado e os canteiros para o plantio da alface americana e do repolho feitos manualmente. As hortaliças foram transplantadas em maio de 2002, sendo o espaçamento de $0,50 \times 0,30 \mathrm{~m}$ para a alface americana, e o repolho em covas, com espaçamento de $0,60 \times 0,40 \mathrm{~m}$. Duas linhas úteis para a alface americana e três para o repolho foram consideradas nas avaliações.

$\mathrm{Na}$ testemunha, utilizou-se adubação química, ou seja, o cultivo convencional, baseada na análise de solo e na recomendação para as culturas de acordo com a Comissão de Fertilidade do Solo do Estado de Minas Gerais (1999). Para a alface americana, foram empregados $400 \mathrm{~kg} \cdot \mathrm{ha}^{-1} \mathrm{de}$ $\mathrm{P}_{2} \mathrm{O}_{5}, 150 \mathrm{~kg} \cdot \mathrm{ha}^{-1}$ de $\mathrm{K}_{2} \mathrm{O}$ e $150 \mathrm{~kg} \cdot \mathrm{ha}^{-1}$ de N. O nitrogênio foi parcelado em $75 \mathrm{~kg} \cdot \mathrm{ha}^{-1}$ no plantio, $50 \mathrm{~kg} \cdot \mathrm{ha}^{-1}$ aos 30 dias após o plantio e $25 \mathrm{~kg} \mathrm{ha}^{-1}$ aos 45 dias após o plantio. Para o repolho, utilizaram-se $100 \mathrm{~kg} \cdot \mathrm{ha}^{-1}$ de $\mathrm{P}_{2} \mathrm{O}_{5}, 100 \mathrm{~kg} \cdot \mathrm{ha}^{-1} \mathrm{de}$ $\mathrm{K}_{2} \mathrm{O}$ e $150 \mathrm{~kg} \cdot \mathrm{ha}^{-1}$ de N. O nitrogênio foi parcelado em 30 kg.ha- ${ }^{-1}$ no plantio e aos 30 dias após o plantio e $45 \mathrm{~kg}$.ha${ }^{1}$ aplicados aos 45 e 65 dias após o plantio. Também se aplicou $1 \mathrm{~g}$ de ácido bórico por cova. Nas parcelas com as leguminosas utilizou-se a adubação com composto orgânico na dosagem de 20 t.ha $^{-1}$ (peso úmido), em duas aplicações, 10 t.ha $^{-1}$ no plantio e 10 t.ha $^{-1} 30$ dias após o plantio, para ambas as hortaliças.

As hortaliças foram irrigadas por aspersão com lâmina média de $8,70 \mathrm{~mm} \cdot \mathrm{dia}^{-1}$ e CUC $78 \%$ (Coeficiente de uniformidade de Christiansen). A alface americana e o repolho foram colhidos aos 65 e 95 dias após o transplantio, respectivamente.

Para avaliar a população de nematóides na área experimental, onde se encontrava alta densidade populacional de fitonematóides, foram retiradas cinco amostras simples de solo por parcela na profundidade de $20 \mathrm{~cm}$. As amostras foram retiradas aos 45 dias após o plantio das leguminosas, e aos 30, 60 e 90 dias após o plantio das hortaliças. De cada amostra retiraram-se $100 \mathrm{~cm}^{3}$ de solo para realizar a extração dos nematóides por flotação e centrifugação em solução de sacarose (Jenkins, 1964).

As espécies de nematóides avaliadas no experimento foram Helicotylenchus dihystera (Cobb) Sher., Meloidogyne javanica (Treub) Chitw. e M. incognita (Kofoid \& White) Chitw. Apesar da pouca importância econômica de $H$. dihystera, este foi quantificado para verificar a possível eficiência da adubação verde na redução de fitonematóides ectopararitos. As espécies de Meloidogyne foramidentificadas pela técnica do corte perineal, porém para a quantificação, as espécies não foram diferenciadas em um único grupo. Os dados de contagem das espécies H. dihystera e Meloidogyne spp. foram transformados utilizando-se a equação $\sqrt{\mathrm{x}}$ por não haver homogeneidade de variância dos erros.

Não houve interação significativa entre as hortaliças e a adubação verde para a população de Meloidogyne spp, ou seja, não houve diferenças, no cultivo orgânico, entre a alface e o repolho. De acordo com o teste de médias, a 
densidade populacional de nematóides aos 45 dias após o plantio das leguminosas apresentou diferença significativa apenas para Meloidogyne spp. (M. incognita e M. javanica). Nas parcelas cultivadas com mucuna-preta observou-se menor número de juvenis de Meloidogyne no solo (Tabela 1). As espécies de Meloidogyne, no tratamento com vegetação espontânea, provavelmente, encontraram hospedeiro favorável à sua multiplicação. Dados semelhantes foram obtidos com a incorporação da parte aérea de Crotalaria paulinia Schrank, controlando eficientemente a reprodução de $M$. incognita raça 3 no sistema radicular do feijoeiro (Gonzaga \& Ferraz, 1994), porém em cultivo convencional. $\mathrm{Na}$ cultura da cenoura (Daucus carota L.), plantada 120 dias após a eliminação manual das plantas antagonistas Tagetes erecta L., Crotalaria spectabilis, Centrosema pubescens Benth., Pueraria javanica Benth. e Stylosanthes guyanensis Sw. pode-se verificar o declínio populacional de M. incognita raça $1 \mathrm{em}$ todas as parcelas, exceto as plantadas com P. javanica e Abelmoschus esculentes, cultura utilizada como testemunha devido à alta suscetibilidade ao nematóide (Charchar \& Vieira, 1991).

Nas demais avaliações, aos 30, 60 e 90 dias após o plantio das hortaliças, $H$. dihystera apresentou diferença significativa para a interação hortaliças x plantas de cobertura aos 60 dias, e para o efeito do fator plantas de cobertura, isoladamente, aos 30 e 90 dias após plantio das hortaliças. Aos 30 e 90 dias, observou-se menor população de $H$. dihystera nas parcelas incorporadas com feijão-de-porco, independente das hortaliças (Tabela 2). Vale ressaltar que, aos 90 dias, a alface americana já havia sido colhida e os dados se referem somente ao repolho. Em estudos com mucuna-preta, mucunabranca [Mucuna nivea (Roxb.) D.C.], Crotalaria juncea, Crotalaria spectabilis, arroz (Oryza sativa L.), milho (Zea mays L.) e caupi [Vigna unguiculata (L.) Walp.], plantados em vasos com adubação química, verificou-se redução no número de Helicotylenchus multicinctus (Cobb) Golden. no solo e raízes em plantas de arroz, mucuna-preta, mucunabranca, C. juncea e C. spectabilis (Bringel \& Silva, 2000), todos em cultivo convencional, empregando-se adubação e o controle de pragas e doenças da parte aérea.

Aos 60 dias após o plantio das hortaliças, observouse, nas áreas incorporadas com crotalária, menor número de $H$. dihystera nas parcelas cultivadas com repolho, enquanto nas áreas cultivadas com alface americana a densidade populacional foi igual para todas as parcelas (Tabela 3). Moura et al. (1997), no plantio em campo, de Crotalaria juncea e mucuna-preta seguida pelo cultivo do amendoim (Arachis hypogaea L.), não observaram diferenças no nível populacional de $H$. dihystera. Dessa forma, demonstrouse que o uso de adubação verde como tática de controle de fitonematóides pode ser viável para alguns patossistemas em cultivo orgânico e deve ser integrado a alternativas de manejo como o uso de variedades resistentes, solarização e aração seguida de irrigação do solo, principalmente no cultivo orgânico.
TABELA 1 - População de Meloidogyne spp. em função das plantas de cobertura aos 45 dias após semeio das leguminosas ${ }^{1}$. UFLA, Lavras, MG, 2002

\begin{tabular}{|c|c|}
\hline Plantas de cobertura & $\begin{array}{l}\text { Número de J2 de Meloidogyne spp./ } \\
100 \text { ce de solo }\end{array}$ \\
\hline Mucuna -preta & $1,23 \quad \mathrm{c}$ \\
\hline Crotalari a juncea & $2,06 \quad b \quad c$ \\
\hline Feijão-de-porco & 5,60 a b \\
\hline Vegetação espontânea & $6,77 \mathrm{a}$ \\
\hline CV $(\%)$ & 45,03 \\
\hline
\end{tabular}

TABELA 2 - População de Helicotylenchus dihystera em função das leguminosas e vegetação espontânea aos 30 e 90 dias após plantio da alface americana e do repolho ${ }^{1}$

\begin{tabular}{|c|c|c|}
\hline \multirow[t]{2}{*}{ Plantas de cobertura } & \multicolumn{2}{|c|}{$\begin{array}{c}\text { População de Helicotylenchus } \\
\text { dihystera /100ce de solo }\end{array}$} \\
\hline & $30 \operatorname{dias}^{1}$ & 90 dias $^{1,2}$ \\
\hline Feijão-de-porco & $16,80 \quad b$ & $9,47 \quad b$ \\
\hline Mucuna -preta & $52,67 \mathrm{a} \quad \mathrm{b}$ & $77,20 \mathrm{a}$ \\
\hline Crotalária juncea & $61,14 \mathrm{a}$ & $67,20 \mathrm{a}$ \\
\hline Vegetação espontânea & $33,34 \mathrm{a} \quad \mathrm{b}$ & $32,76 \mathrm{a} \quad \mathrm{b}$ \\
\hline $\mathrm{CV}(\%)$ & 32,47 & 24,29 \\
\hline
\end{tabular}

${ }^{1}$ Médias seguidas das mesmas letras na coluna não diferem entre si pelo teste de Tukey, a $5 \%$ de probabilidade.

${ }^{2}$ Médias da população de Helicotylenchus dihystera em solo cultivado com repolho.

TABELA 3 - População de Helicotylenchus dihystera em função das hortaliças e plantas de cobertura aos 60 dias após o plantio da alface americana e do repolho ${ }^{1}$

\begin{tabular}{llc}
\hline \hline \multirow{2}{*}{ Plantas de cobertura } & \multicolumn{2}{c}{$\begin{array}{c}\text { População de Helicotylenchus } \\
\text { dihystera /100cc de solo }\end{array}$} \\
\cline { 2 - 3 } & \multicolumn{1}{c}{ Repolho } & Alface americana \\
\hline Crotalaria juncea & 10,27 b B & 61,34 a A \\
Mucuna -preta & 36,40 a A & 20,53 a A \\
Feijão-de-porco & 31,60 a A & 10,40 a A \\
Vegetação espontânea & 32,01 a A & 30,93 a A \\
\hline CV(\%) & 35,85 & 19,63 \\
\hline
\end{tabular}

${ }^{1}$ Médias seguidas das mesmas letras minúsculas na linha e maiúsculas na coluna não diferem entre si pelo teste de Tukey, a 5\% de probabilidade.

\section{REFERÊNCIAS BIBLIOGRÁFICAS}

ALCANTÂRA, F.A., NETO, A.E.F., PAULA, M.B., MESQUITA, H.A. \& MUNIZ, J.A. Adubação verde na recuperação da fertilidade de um latossolo vermelho-escuro degradado. Pesquisa Agropecuária Brasileira 35:277-288. 2000.

ANDRADE, N.C. \& PONTE, J.J. Efeito do sistema de plantio em camalhões e do consórcio com Crotalaria spectabilis no controle de Meloidogyne incognita em quiabeiro. Nematologia Brasileira 23:11-16. 1999.

BRINGEL, J.M.M. \& SILVA, G.S. Efeito antagônico de algumas espécies de plantas a Helicotylenchus multicinctus. Nematologia Brasileira 24:179-181. 2000. 
CHARCHAR, J.M. \& VIEIRA, J.V. Controle de Meloidogyne incognita raça 1 em cenoura cv. 'Nantes' através de rotação com plantas antagônicas. Fitopatologia Brasileira 16:196-199. 1991.

COMISSÃO DE FERTILIDADE DO SOLO DO ESTADO DE MINAS GERAIS. Recomendações para o uso de corretivos e fertilizantes em Minas Gerais: 5a aproximação. 2. ed. Viçosa. 1999.

DAROLT, M.R. Agricultura orgânica: inventando o futuro. Londrina PR. IAPAR. 2002.

GONZAGA, V. \& FERRAZ, S. Efeito da incorporação da parte aérea de algumas espécies vegetais no controle de Meloidogyne incognita Raça 3. Nematologia Brasileira 18:42-49. 1994.

JENKINS, W.R. A rapid centrifugal-flotation technique for separating nematodes from soil. Plant Disease Reporter 48:692. 1964.

MOURA, R.M., MOURA, A.M., MACEDO, M.E.A. \& SILVA, E.G. Influência de três diferentes combinações de culturas sobre populações de nematóides associados à cana-de-açúcar. Nematologia Brasileira 21:75-83. 1997.

PEREIRA, J., BURLE, M.L. \& RESCK, D.V.S. Adubos verdes e sua utilização no cerrado. Anais. Simpósio sobre Manejo e Conservação do Solo no Cerrado, Goiânia, GO. Campinas SP. Fundação Cargil. 1992. pp. 140-154.

RIBAS, R.G.T., JUNQUEIRA, R.M., OLIVEIRA, F.L., GUERRA, J.G.M., ALMEIDA, D.L. \& RIBEIRO, R.L.D. Adubação verde na forma de consórcio no cultivo do quiabeiro sob manejo orgânico. Comunicado Técnico 54. 2002.

YUSSEFI, M. \& WILLER, H. (Org.). The world of organic agriculture statistics and future prospects, 2003. www.soel.de/ inhalte/publikationen/s/s_74.pdf.

ZAMBOLIM, L., VALE, F.X.R. \& COSTA, H. Controle de doenças de plantas - Hortaliças. v. 2. Viçosa MG. Suprema Gráfica e Editora. 2000. 